\title{
CONVERGENCE OF PROBABILITY MEASURES ON SEPARABLE BANACH SPACES
}

\author{
L. S. GRINBLAT
}

\begin{abstract}
The following result follows immediately from a general theorem on the convergence of probability measures on separable Banach spaces: On the space $C[0,1]$ there exists a norm $p(x)$ equivalent to the ordinary norm such that if $\xi_{1}(t), \ldots, \xi_{n}(t), \ldots$ and $\xi(t)$ are continuous random processes $(0<t<1)$ and for any finite set of points $t_{1}, \ldots, t_{k} \subset[0,1]$ the joint distribution of $p\left(\xi_{n}\right), \xi_{n}\left(t_{1}\right), \ldots, \xi_{n}\left(t_{k}\right)$ converges to the joint distribution of $p(\xi), \xi\left(t_{1}\right), \ldots, \xi\left(t_{k}\right)$, then $\xi_{n}(t)$ converges weakly to $\xi(t)$.
\end{abstract}

1. Let $X$ be a Banach space and $X^{*}$ be the dual space of $X$.

Definition [1, §2]. We say that $X$ has the $H$-property relative to a set $\Gamma \subset X^{*}$ if from the conditions $\left\|x_{n}\right\|=\|x\|=1, \lim _{n \rightarrow \infty} f\left(x_{n}\right)=f(x)$ for all $f \in \Gamma$ it follows that $\lim _{n \rightarrow \infty}\left\|x_{n}-x\right\|=0$.

In what follows we replace the phrase, " $X$ has the property $H$ relative to $\Gamma$ ", by the symbol $X \in H_{\Gamma}$.

THEOREM 1 (KADETS, SEE $[1, \S 2])$. Let $X$ be a separable Banach space, and $\Gamma$ be a subset of $X^{*}$ such that

$$
\inf _{x \in X} \sup _{f \in \Gamma} \frac{|f(x)|}{\|f\| \cdot\|x\|}=\eta(\Gamma)>0 \quad(x \neq 0, f \neq 0) .
$$

Then we can introduce in $X$ an equivalent norm $h(x)$ relative to which $X \in H_{\Gamma}$.

It is easy to see that if $\eta(\Gamma)>0$, then there exists a countable set $\Gamma_{0} \subset \Gamma$ such that $\eta\left(\Gamma_{0}\right)>0$.

In what follows in this section, we suppose that $X, \Gamma, h(x)$ satisfy the conditions of Theorem 1 and $\Gamma$ is a countable set.

Let

$$
S=\{x \in X: h(x)=1\}, \quad B=\{x \in X: h(x) \leqslant 1\} .
$$

We denote by $L_{T}(S)$ the Banach algebra ${ }^{1}$ of bounded continuous functionals, ${ }^{2}$ defined on $S$, which is generated by all functionals from $\Gamma$, restricted to $S$.

Received by the editors September 9, 1976 and, in revised form, March 23, 1977. AMS (MOS) subject classifications (1970). Primary 60B05, 60B10; Secondary 46B99, 54D35. ${ }^{1}$ Throughout this paper, an algebra will mean an algebra with identity.

${ }^{2}$ Throughout this paper, a functional defined on a set $A$ will mean a real function (without supplementary conditions) on $A$. 
We denote by $M_{\Gamma}(B)$ the Banach algebra of bounded continuous functionals, defined on $B$, which is generated by all functionals from $\Gamma$, restricted to $B$, and the functional $h(x)$, restricted to $B$.

LEMMA 1. For any point $x_{0} \in S$ and for any closed set $F \subset S\left(x_{0} \notin F\right)$ there exists $f \in L_{\Gamma}(S)$ such that $f\left(x_{0}\right)=0$ and $f(x) \geqslant 1$ if $x \in F$.

Proof. Let $\Gamma=\left\{f_{1}, \ldots, f_{k}, \ldots\right\}$. Consider numbers $\varepsilon_{1}>\varepsilon_{2}>\ldots>$ $\varepsilon_{k} \ldots$, such that $\varepsilon_{k} \rightarrow 0$. Let $U_{k}=\left\{x \in S:\left|f_{i}\left(x_{0}\right)-f_{i}(x)\right|<\varepsilon_{k}\right.$, $i=$ $1, \ldots, k\}$. There exists $k$ such that $U_{k} \cap F=\varnothing$. Define the functional $f$ by

$$
f(x)=\frac{1}{\varepsilon_{k}^{2}} \sum_{i=1}^{k}\left(f_{i}\left(x_{0}\right)-f_{i}(x)\right)^{2} \text {. Q.E.D. }
$$

LEMMA 2. For any point $x_{0} \in B$ and for any closed set $F \subset B\left(x_{0} \notin F\right)$ there exists $f \in M_{\Gamma}(B)$ such that $f\left(x_{0}\right)=0$ and $f(x) \geqslant 1$ if $x \in F$.

Proof. Let $h\left(x_{0}\right)=a$. There exists a positive number $\varepsilon<a$ such that

$$
U=\left\{x \in B:|h(x)-a|<\varepsilon, h\left(x a / h(x)-x_{0}\right)<\varepsilon\right\}
$$

is contained in $B \backslash F$. Let

$$
\hat{F}=\{x \in B \backslash U: h(x)=a\} .
$$

By Lemma 1 applied to $\hat{F}$ there exists $\hat{f} \in M_{\Gamma}(B)$ such that $\hat{f}\left(x_{0}\right)=0$ and $\hat{f} \geqslant 1$ on $\hat{F}$. Since functionals in $M_{\Gamma}(B)$ are uniformly continuous, there exists a positive number $\delta<\min \left(\varepsilon, \frac{1}{2}\right)$ such that $\hat{f}(x)>\frac{1}{2}$ if $|h(x)-a|<\delta$ and $x a / h(x) \in B \backslash U$. If $x \in F$, either $|h(x)-a|>\delta$, or $|h(x)-a|<\delta$ and $x a / h(x) \in B \backslash U$. Therefore we may take $f$ to be

$$
f(x)=\frac{1}{\delta^{2}}\left[\hat{f}(x)^{2}+(h(x)-a)^{2}\right] . \quad \text { Q.E.D. }
$$

In $[2, \S 5]$ and in $[3, \S 2]$ the following was proved:

LEMMA 3. Let the probability measures $\mu_{1}, \ldots, \mu_{n}, \ldots$ and $\mu$ be defined on the Borel sets of the separable metric space $\Omega$ and let $G(\Omega)$ be a Banach algebra of bounded continuous functions defined on $\Omega$. Suppose that for any point $\omega_{0} \in \Omega$ and for any closed set $F \subset \Omega\left(\omega_{0} \notin F\right)$ there exists $g \in G(\Omega)$ such that $g\left(\omega_{0}\right)=0$ and $g(\omega) \geqslant 1$ for all $\omega \in F$. Suppose, finally, that for every function $g \in G(\Omega)$ the distribution of $g(\omega)$ with respect to the measure $\mu_{n}$ converges to the distribution of $g(\omega)$ with respect to the measure $\mu$. Then $\mu_{n}$ converges weakly to $\mu$.

Lemmas 2 and 3 imply the following:

THEOREM 2. Let the probability measures $\mu_{1}, \ldots, \mu_{n}, \ldots$ and $\mu$ be defined on the Borel sets of $X$. If for any finite set of functionals $f_{1}, \ldots, f_{k} \subset \Gamma$ the joint distribution of $h(x), f_{1}(x), \ldots, f_{k}(x)$ with respect to the measure $\mu_{n}$ converges to the joint distribution $h(x), f_{1}(x), \ldots, f_{k}(x)$ with respect to the measure $\mu$, then $\mu_{n}$ converges weakly to $\mu$. 
2. Consider the space $C[0,1]$ with the ordinary norm $\|x(t)\|=$ $\max _{0<t<1}|x(t)|$. Let $\Gamma$ be the set of functionals $f(x)=x(\tau)$, where $\tau$ is rational, $0 \leqslant \tau \leqslant 1, x \in C[0,1]$. It is obvious that $\eta(\Gamma)=1$. By virtue of Theorem 1 on the space $C[0,1]$ there exists a norm $p(x)$, equivalent to the ordinary norm, relative to which $C[0,1] \in H_{\Gamma}$. Theorem 2 implies the following:

THEOREM 3. Let $\xi_{1}(t), \ldots, \xi_{n}(t), \ldots$, and $\xi(t)$ be continuous random processes. If for any finite set of points $t_{1}, \ldots, t_{k} \subset[0,1]$ the joint distribution of $p\left(\xi_{n}\right), \xi_{n}\left(t_{1}\right), \ldots, \xi_{n}\left(t_{k}\right)$ converges to the joint distribution of $p(\xi), \xi\left(t_{1}\right), \ldots, \xi\left(t_{k}\right)$, then for any continuous functional $f$ on $C[0,1]$ the distribution of $f\left(\xi_{n}\right)$ converges to the distribution of $f(\xi)$.

One can give an explicit formula for the norm $p(x)$. For example

$$
p(x)=\max _{0<t<1}|x(t)|+\sum_{n=1}^{\infty} \frac{1}{2^{n}} \cdot \max _{|t-s|<1 / n}|x(t)-x(s)| .
$$

3. It is obvious that if we weaken the hypothesis of Theorem 3 , and demand only that the joint distribution of $\xi_{n}\left(t_{1}\right), \ldots, \xi_{n}\left(t_{k}\right)$ converges to the joint distribution of $\xi\left(t_{1}\right), \ldots, \xi\left(t_{k}\right)$, then, in general, the conclusion no longer holds. Therefore, it is interesting to compare Theorem 3 to the following results:

THeOREM 4 [4]. Let $\xi_{1}(t), \ldots, \xi_{n}(t), \ldots$, and $\xi(t)$ be measurable random processes $(0 \leqslant t \leqslant 1)$. Suppose that there exist $C$ and $p \geqslant 1$ such that for all $n$ and $t$ we have $E\left|\xi_{n}(t)\right|^{p} \leqslant C$. Suppose, finally, that for any finite set of points $t_{1}, \ldots, t_{k} \subset[0,1]$ the joint distribution of $\xi_{n}\left(t_{1}\right), \ldots, \xi_{n}\left(t_{k}\right)$ converges to the joint distribution of $\xi\left(t_{1}\right), \ldots, \xi\left(t_{k}\right)$ and $E\left|\xi_{n}(t)\right|^{p} \rightarrow E|\xi(t)|^{p}$ for all $t \in[0,1]$. Then for any continuous functional $f$ on $L_{p}[0,1]$ the distribution of $f\left(\xi_{n}\right)$ converges to the distribution of $f(\xi)$.

\section{REFERENCES}

1. V. D. Mil'man, Geometric theory of Banach spaces. II, Russian Math. Surveys 26 (1971), no. 6, 79-163.

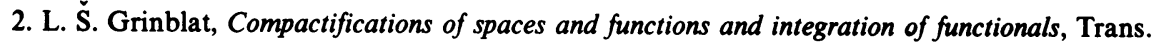
Amer. Math. Soc. 217 (1976), 195-2

3. Convergence of random processes without discontinuities of the second kind and limit theorems for sums of independent random variables, Trans. Amer. Math. Soc. (to appear).

4. Math. Soc. 61 (1976), 371-376.

Department of Mathematics, Bar-Illan University, Ramat-Gan, Israel

Current address: Department of Mathematics, Harvard University, Cambridge, Massachusetts 02138 